\title{
LOS APODOS EN LA EDUCACIÓN SUPERIOR COLOMBIANA
}

\section{THE NICKNAMES IN COLOMBIAN HIGHER EDUCATION}

\author{
Nevis Balanta Castilla ${ }^{1}$ \\ Universidad Distrital de Bogotá
}

\section{RESUMEN}

Este artículo se centra en torno a la construcción de imaginarios y representaciones sobre los docentes por parte de estudiantes universitarios, quienes mediante la metaforización expresan opiniones compartidas sobre el quehacer pedagógico y educativo de sus profesores e incluso sobre su comportamiento en la cotidianidad. Se ofrece además un repertorio de apodos de maestros colocados por jóvenes universitarios, quienes entre otras estrategias semánticas, utilizan la metáfora como eje central para nombrar a sus profesores.

$1 \quad$ Lic. Lingüística y literatura. Magíster en Investigación Social. Universidad Distrital Francisco José de Caldas. Estudios de periodismo de la Universidad Estatal de Voronezh-Rusia. Docente adscrita a la Facultad Tecnológica.. Directora de Grupo de Investigación: Lenguaje y Tecnología(LENTE) y del semillero Étymos de la Facultad tecnológica de Universidad Distrital de Bogotá. nbalantac@udistrital.edu.co

Orcid: 0000-0003-1502-290
El texto comprende además elementos de tipo descriptivo y explicativo acerca de la acción de apodar en un contexto académico. Asimismo se destacan los recursos sintácticos y semánticos de los que se vale el estudiante apodador para crear el nuevo nombre y se habla de las motivaciones y características del apodo en su proceso de formación, un tema novedoso e interesante en el ámbito de la investigación educativa. La investigación es sociodialectal, pues se aborda una parte de la lengua y se intenta establecer relaciones entre lo lingüístico y lo sociocultural. Para ello se recopiló un corpus de 700 apodos a través de encuestas y entrevistas realizadas a 2000 estudiantes entre 16 y 35 años. 
PALABRAS CLAVES: metáfora, apodos, imaginarios y representaciones.

\section{ABSTRACT}

This article focuses on the construction of imaginaries and representations about teachers by university students, who through metaphorization express shared views on the pedagogical and educational work of their teachers and even on their behaviour in everyday life. It also offers a repertoire of teachers' nicknames placed by young university students, who, among other semantic strategies, use the metaphor as the central axis to appoint their professors. The text also includes descriptive and explanatory elements about the action of nicking in an academic context. Also highlights the syntactic resources.

The text also includes descriptive and explanatory elements about the action of nicking in an academic context. It also highlights the syntactic and semantic resources that the student nickname uses to create the new name and talks about the motivations and characteristics of the nickname in its formation process, a novel and interesting topic in the field of educational research. The research is sociodialectal, since it deals with a part of the language and tries to establish relations between the linguistic and the sociocultural. To do this, a corpus of 700 nicknames was collected through surveys and interviews with 2000 students between the ages of 16 and 35 .

KEYWORDS: metaphor, nicknames, imaginaries and representations.

\section{DEFINICIÓN}

El apodo es un tipo de representación nominal que se hace respecto al sujeto-maestro, del cual se resaltan defectos físicos o actitudinales, o de manera contraria se elogian cualidades. La palabra apodo procede de la palabra latina apputare que significa juzgar, evaluar y comparar. El apodo es además un acto de creación y recreación lingüística o en palabras de AUSTIN un acto de habla motivado mediante el cual el sujeto apodador da un nuevo nombre a su profesor según las características que evocan en la mente del estudiante la imagen de un objeto, personaje o circunstancia que recibe este nuevo nombre.

Hay muchas razones que llevan al estudiante a apodar a su maestro: jocosidad, rabia, ira, cariño y maldad.

El apodo para muchos es la patología del nombre pero más allá de esto, hay que resaltar que éste es un tema tan antiguo como el hombre mismo. En las tribus primitivas los hombres usaban apodos como signo de conocimiento distintivo. En Roma el agnomen o apodo era importantísimo para los guerreros y para los griegos fue tan relevante que algunos filósofos son más conocidos por el apodo que por su verdadero nombre, caso de PLATÓN que significa ancho de hombros, aunque su verdadero nombre es Aristocles. Aquiles significa sin labio y Telémaco el que lucha lejos.

El apodo tampoco escapa a los diferentes ámbitos como el deporte, la política, la Internet y la cultura en general. En el deporte son conocidos: Cochise Rodríguez, el Pibe Valderrama, el Tren Valencia, Pelé, el Tino, entre otros. En la política el ex presidente López fue apodado el pollo López y en el conflicto colombiano son de uso común apodos como Tirofijo, Mono Jojoy y Jorge 40.

De manera entonces que como no es un tema exclusivo de una época o de una generación, lo que importa en esta investigación es el contexto en el que surgen los apodos y las condiciones socioculturales e intenciones de los estudiantes para apodar a sus profesores. 


\section{PRECISIONES CONCEPTUALES:}

En ese orden de ideas para entender mejor el tema de los apodos es importante hacer algunas precisiones y diferencias entre algunos conceptos que tienden a confundirse:

Nombre: palabra con que son designados los objetos, las personas, los animales y las cosas. El nombre es tan importante que como dice Borges:"Si como el griego afirma en el Cratilo/ el nombre es arquetipo de la cosa/ en el nombre de Rosa está la rosa/ y todo el Nilo en la palabra Nilo...".

Sobrenombre: nombre que se añade a veces al apellido o nombre para distinguir a dos o más personas que tienen el mismo.

Seudónimo: nombre empleado por escritores o artistas en vez del propio o nombre de pila. Ejemplo: Pablo Neruda en vez de Neftalí Ricardo Reyes o Rubén Darío por Félix Rubén García Sarmiento.

Hipocorístico: nombre cariñoso, diminutivo afectuoso. Se utiliza eufemísticamente para demostrar simpatía, amistad o amor. Algunos ejemplos son: Sandrita por Sandra, Olguita por Olga, Gusta por Gustavo Pepe por José, Pato en vez de Patricia, Davo por David y Tami por Tamara.

Alias: nombre que se coloca a los delincuentes en el mundo del hampa. Ej:

\section{$>\quad$ El Patrón a Pablo Escobar por sumisión $y$ respeto.}

> Grannobles a Germán Briceño, por un líder francés de la $2^{\mathrm{a}}$ guerra mundial.

D Las Marías: banda de mujeres que robaban supermercados

> Rasguño por Hernando Gómez Bustamante, debido a una cicatriz en la mejilla por efecto del roce de un disparo.

> Mono Jojoy por Jorge Briceño Suárez, llamado así por el gusano jojoy.

$>\quad$ Triple Cero a Salvatore Mancuso, en alusión al código de los radio operadores que asociaban soles de los generales con los ceros en altos rangos de los paramilitares.

Apodo: nombre que se da a una persona teniendo en cuenta su aspecto físico, sus actitudes o comportamiento. Sus sinónimos son mote y remoquete.

Hechas estas aclaraciones pasaremos a dar algunos ejemplos de los apodos recogidos en nuestro corpus, teniendo en cuenta las diferentes rutas que escoge el joven apodador para nombrar a su docente. Una de esa ruta es la distorsión fonética mediante la cual el estudiante juega con los sonidos del apellido o nombre para crear un efecto de sentido, ya sea humorístico, irónico o de rechazo, tal como se aprecia en la siguiente tabla:

\begin{tabular}{|l|l|}
\hline \multicolumn{1}{|c|}{ NOMBRE } & \multicolumn{1}{c|}{ APODO } \\
\hline Edgar & Edgargajo \\
\hline Hildebrando & Integrando \\
\hline Hermes & Herpes \\
\hline Rosendo APELLIDO & Rosquendo \\
\hline \hline \multicolumn{1}{|c|}{ APODO } \\
\hline Olea & \\
\hline Zarta & Oblea \\
\hline Vargas & Enzarta \\
\hline Tangarife & Vergas \\
\hline Valencia & Tanga \\
\hline Melocotón & Falencia \\
\hline Fajardo & Melo \\
\hline Garavito & Pajardo \\
\hline & Garabato \\
\hline
\end{tabular}


La segunda ruta es la morfo-sintáctica.

Contiene los apodos que se realizan por derivación y composición. En el primer caso están los apodos por derivación por prefijos, diminutivos, aumentativos y despectivos. He aquí algunos ejemplos:

\begin{tabular}{|c|c|c|c|}
\hline Apodos por prefijos & $\begin{array}{c}\text { Apodos por } \\
\text { aumentativos }\end{array}$ & $\begin{array}{c}\text { Apodos por } \\
\text { diminutivos }\end{array}$ & Apodos despectivos \\
\hline Superabuela & Cachetona & Masturbín & Menudencias \\
\hline Milivoltio & Cotizón & Fiscalito & Vómito \\
\hline Repollito & Libretón & Copito & Bacute \\
\hline & Muñecón & Pecaditos & Tetanic \\
\hline
\end{tabular}

Los apodos por composición, por su parte, pueden ser producto de las siguientes combinaciones:

Sustantivo más preposición más sustantivo: ronco de oro; ojos de sapo. Sustantivo más adjetivo: Flema suelta; Gorra vieja.

Sustantivo más sustantivo: Doña Tetas.

Verbo más sustantivo: Puyanube.

Adjetivo más sustantivo: Sietelochas, Cuatronalgas.

Adjetivo más adjetivo: Pilotonto

En la ruta morfosintáctica también se encuentra los apodos realizados por onomatopeyas, sustantivos y adjetivos. Entre los primeros están: Tá tá tá; Tilín tilín; La Múuu; Ukis; Pío pío. En los segundos, tenemos: Manguera, Conejo, Diablo, Medusa, Autista, Veneno. Y en los últimos, encontramos los siguientes: Despistado, Gorda, Infame, Loco, Gomelo, Antropófago, Vago, Hipervertido.

También por vía semántica se encuentra, encontramos los apodos que se forman en un proceso de metaforización en el que son explícitos la intención, el contexto y el modo semántico que adquiere la metáfora. En la intención, según las respuestas de los jóvenes, es desahogar su rabia; por el contexto en que surgen los apodos, es el académico, pero asumiendo el habla un carácter marcadamente popular; y en el modo semántico de los apodos se encuentran también hipérboles, metonimias, sinécdoques, símiles, ironías y eufemismos. La tabla que sigue es ilustrativa de lo que se dice.

\begin{tabular}{|l|l|}
\hline \multicolumn{1}{|c|}{ APODOS POR METAFORIZACION } & \multicolumn{1}{c|}{ EXPLICACION } \\
\hline EI duende & Porque aparece y desaparece por arte de magia \\
\hline La pulga & Porque pasa de perra en perra \\
\hline Baygón & Nunca falla \\
\hline Orbitel & Porque no pasa del cero cinco \\
\hline El dólar & Porque sube y baja \\
\hline Árbol de navidad & Siempre está prendido \\
\hline Semana Santa & Porque es larga y sin carnes \\
\hline
\end{tabular}

En este proceso se da una traslación de significados donde hay un elemento $X$ que se compara (sujeto docente), un elemento $\mathrm{Y} u$ objeto comparado (duende, dólar, etc.), y un elemento Z, o apodo, resultante de la relación entre los dos anteriores. 
Además de lo anterior otros ejemplos de apodo se agruparon por temas, o áreas léxicas (concepto de Emma Sopeña). Estas son: aspectos físicos, aspectos académico-pedagógicos, aspectos actitudinales, aspectos estéticos, aspectos de procedencia, aspectos del mundo animal, aspectos del mundo gastronómico y aspectos del mundo mediático.

Aspectos físicos: pinocho, enano, plumablanca, chocorramo, carae'papa...

Aspecto académico-pedagógicos: cuchilla, anestesiólogo, escuelero, Atila, Tumbanotas, Traganúmeros...

Aspectos estéticos: trapitos, carnaval, barrilete...

Aspectos de procedencia: calimeño, cuba, boyacoman, costeño, ruso, pastusito...

Aspectos del mundo animal: ratona, la ballena, galloclaudio, el simio, vaca...

Aspectos gastronómicos: limón, papasalada, cebollita, macarrón, barbiquieu...

Aspectos mediáticos: chapulín, sayayín, misterbín, cantinflas, picacho, teletubi, piolín, agente86, Don Ramón, Condorito, Olafo, Flanders, Milhouse, Topoyiyo...

Observando los ejemplos hay muchos que se hacen por transposición de significados, pues como se sabe las metáforas no son exclusivas del lenguaje literario, sino que también son propias del habla popular y cotidiana. Además desde la perspectiva de Aguiar E Silva la literatura es además una forma de mensaje verbal y su lenguaje es connotativo y plurisignificativo. En este sentido la diferencia que hay entre nombre $y$ apodo es que el primero es denotativo y el segundo connotativo.

La metáfora impregna entonces el pensamiento y el lenguaje de los jóvenes y sirve de mecanismo para expresar inconformismos en el proceso de aprendizaje. La selección de los objetos que se comparan, base de las metáforas, emergidas en el apodo son conceptos, imaginarios $y$ representaciones albergadas en la mente de los jóvenes y ancladas en su cultura.(Navarro y Balanta, 2019)

\section{MOTIVACIONES Y CARACTERÍSTICAS DEL APODO:}

El apodo, como se ha visto, tiene motivaciones y características que le son inherentes. Las motivaciones pueden ser comunicativas, sicológicas y pedagógicas. Son comunicativas cuando hay necesidad de expresar de manera espontáneas opiniones afectivas, sociales, estéticas y morales. Es la vieja necesidad de que hablara Platón en el Cratilo, en el sentido de que es natural del hombre nombrar las cosas.

Son sicológicas en tanto el inconsciente está estructurado como lenguaje y se evidencia o concreta en lo que se dice y se nombra, tal como en su momento lo expresara Lacán, quiere decir esto, que los apodos son actos lingüísticos represados que tienen la necesidad de comunicarse. Tales denominaciones apodísticas son actos que confunden el decir con el hacer, son enérgicos y proclaman el no valor del objeto denominado (Guiraud: 1985).

Y son pedagógicas las motivaciones en virtud de que los apodos comportan una lección implícita de crítica a aspectos que están estrechamente ligados con las representaciones pedagógicas del profesor objeto del apodo. En este sentido Nietzsche planteó que no se le enseña con lo que se sabe, son con lo que se es, algo cada vez más olvidado y subestimado en la práctica docente universitaria.

Respecto a las características, los apodos encierran una primera que es la de la creatividad. Mariano Lozano (1999) dice sobre este aspecto que se necesita gran recursividad lingüística para la creación de apodos, no tanto en la elaboración de neologismos, sino en la 
asociación semántica entre campos distintos para dar lugar a las metáforas.

Una segunda característica es la del anonimato, pues no se conoce con exactitud qué individuo crea el apodo pero éste se vuelve de uso común de la comunidad y se convierte en representación. Otra característica es la afectividad, la cual refleja necesidades emocionales o privaciones afectivas de quiénes los gestan y se convierten a su vez, en actitudes síquicas que se externalizan nombrándolos. También es propio del apodo, el relajo, una característica además muy latinoamericana, tal como lo afirma el mexicano Jorge Portilla (1986). Esta característica hace parte de ese comportamiento que suspende la seriedad con el objetivo de desplazar la atención a o trivial. Allí hay un cierto carácter de digresión o desvío de algo para distensionar a un grupo.

Unas características más son la caricaturización y el humor, pues en el apodo se conjuga la sátira y el humor y existe la intención de poner en ridículo y criticar a personas que están por encima de la jerarquía social del apodador. Por último se debe registrar la oralidad como característica muy enfática en América Latina, las cual en nuestro caso se acompaña de otros lenguajes complementarios como gestos, mímicas, risotadas.

\section{CONCLUSIÓN:}

Para finalizar, los apodos en general, son representaciones vehiculares del imaginario colectivo. En el caso específico de los apodos a los docentes, se expresan lingüísticamente como opiniones compartidas por la comunidad estudiantil, que reflejan los saberes, creencias y valores sobre sus profesores. El apodo es además una expresión informal que se expande en red, con permanencia en el grupo y duradero en el tiempo. Se conforma así una cadena sólida en la que hay una serie de eslabones que divulgan y transmiten el mensaje, lo incorporan y lo convierten en un nombre que sustituye en no pocos casos, el nombre propio de las personas.

\section{BIBLIOGRAFÍA}

AUSTIN, J.L. (1982). Cómo hacer cosas con palabras. Palabras y acciones. Barcelona. Ediciones Paidos.

ALONSO, M.(1960) Ciencia del lenguaje y arte del estilo. Ed. Aguiar. Madrid, 1960.

BAENA, L. A. (1985). Análisis de los actos de habla. Cali. Mimeo Universidad del Valle.

BEINHAUER, Werner.(1973) El español coloquial. Ed. Gredos, 2a . Ed. Madrid,

BALANTA CASTILLA N,(2008) . Metáforas juveniles y lenguajes emergentes. En: Mundos y narrativas de jóvenes. Bogotá

CASARES, J (1975). Diccionario ideológico de la Lengua Española. Ed. Gustavo Gili. Barcelona,

ATKINSON, P y COFFEY, A.( 2003.) Encontrar el sentido a los datos cualitativos. Estrategias complementarias de investigación. Medellín: Universidad de Antioquia,

COROMINAS, J y PASCUAL, J. A. Diccionario crítico etimológico castellano e hispánico. Ed. Gredos. Madrid, 1984. Diccionario de la Lengua Española. Real Academia Epañola. Ed. Espasa Calpe. Madrid, 1970 (19. $\left.{ }^{a}\right)$ y 1984 (20.. $)$.

COSERIU, E (1989), Teoría del lenguaje $y$ lingüística general, $3^{a}$ ed. revisada y corregida, Madrid, Gredos, Biblioteca Románica Hispánica, no. 61 .

ESCANDELL-VIDALL, M. V (1999), Introducción a la pragmática, Barcelona, Ariel. 
GARCIA DE DIEGO, V. (1973)Lecciones de Lingüistica Española. Ed. Gredos.. $3^{a}$. ed. Madrid,

$\begin{array}{lll}\text { Gran } & \text { Enciclopedia } & \text { Larousse. } \\ (1971) & \text { Ed. } \quad \text { Planera. } & \text { Barcelona, }\end{array}$

HALLIDAY, M.. (1982). El lenguaje como semiótica social. La interpretación social del lenguaje y del significado. México. Fondo de Cultura Económica.

HYMES, Dell. H. (1974). Hacia etnografías de la comunicación. En Antología de estudios de etnolingüística. p.48-89. México, Universidad Autónoma de México. Instituto de Investigaciones Antropológicas.

LAKOFF, G y JOHNSON, M(1980) Metáforas de la vida cotidiana..Madrid: Cátedra.

MORI, O., (1989). "El apodo: procedimientos de creación", en D. Kremer, Actes du XVIII Congres Internacional de Linguistique et de Philologie Romanes, Tübingen, Max Niemeyer Verlag, pp. 598-607.

NAVARRO, David y BALANTA, Nevis.(2019). Educar entre desiguales o el cuento de el gallo capón. Bogotá. Universidad Distrital Francisco José de Caldas.

ORTEGA ARAGON, Gonzalo: "Del apellido al mote en El Diario Palentino", 5-12-1988.

SEARLY, John R. (1986). Actos de habla. Ensayo de filosofía del lenguaje. Madrid, Cátedra.

SCHOPENHAUER, Arthur, (1983) El mundo como voluntad y representación, traducción del alemán por Eduardo Ovejero y Maury, México, Porrúa, Col. "Sepan Cuantos ..." No. 419.

TORRES SÁNCHEZ, Ma. Ángeles (1999), Estudio pragmático del humor, Cádiz, Universidad de Cádiz, Documentos de Investigación
VAN DIJK, Teun. El discurso como estructura y proceso. Barcelona: Gedisa,2000.

El discurso como interacción social. Barcelona: Gedisa,2000.

Ideología.una aproximación multidisciplinaria. Barcelona: Gedisa,2000.

VAL, José Delfín: "Apodos, motes y cognomentos", en Revista Folklore, $n^{\circ}$. 3. Edita Caja Popular de Ahorros de Valladolid, 1981. 